\title{
Islamisches und ethisches Wirtschaftsrecht - Risikoverteilung bei fehlender Vereinbarkeit mit den religiösen oder ethischen Vorgaben
}

\author{
Matthias Casper*
}

A. Islamisches und Ethisches Wirtschaftsrecht - Begriff und Erscheinungsformen ................................ 251

I. Problemaufriss ................... 251

II. Begrifflichkeiten und Bestandsaufnahme

B. Destillation von Rechtsfragen ........ 256

C. Die Kompatibilität mit außerrechtlichen Vorgaben als Vertragsgrundlage?

I. Erfahrungen aus der englischen Gerichtspraxis .................... 257

II. Fehlende Shariakonformität als Vertragsgrundlage? 260

1. Überblick zur Diskussion um die Geschäftsgrundlage im deutschen Recht 260

2. Abgrenzung zur vertraglich übernommenen Risikoverteilung..... 261
3. Mögliche Anwendungsfälle der Geschäftsgrundlage ............. 264

4. Zwischenfazit................. 265

D. Auslegung des Vertrages anhand außerrechtlicher Vorgaben?........... 265

E. Risikoverteilung (die sog. Shariafalle) und Haftung ....................... 267

I. Fallgruppen und Risikoverteilung. .. 267

II. Anfängliches Fehlen der Konformität .............................. 269

III. Nachträglicher Wegfall der Konformität............................ 270

IV. Verstoß gegen Anlagegrundsätze.... 271

V. Die Problematik sog. Haftungsfreizeichnungsklauseln............... 271

F. Zusammenfassendes Fazit............ 273

\section{A. Islamisches und Ethisches Wirtschaftsrecht - Begriff und Erscheinungsformen}

\section{Problemaufriss}

Während des Höhepunktes der 2008 ausgebrochenen Finanzmarktkrise rieb sich auch derjenige verwundert die Augen, der nicht nur den Wirtschaftsteil seiner Zeitung, sondern auch den Osservatore Romano liest. Selbst in diesem Hort römischkatholischer Prinzipienfestigkeit schickten sich zwei Wirtschaftswissenschaftlerinnen an, das islamische Kreditwesen als Fels in der Brandung der weltweit erodierenden konventionellen Finanzwirtschaft zu preisen. ${ }^{1}$ Auf der anderen Seite der Skala blüht nicht erst seit Fukushima der Handel mit ethisch oder ökologisch ausgerich-

* Prof. Dr. Matthias Casper ist Inhaber des Lehrstuhls für Bürgerliches Recht, Gesellschafts-, Bank- und Kapitalmarktrecht am Institut für Unternehmens- und Kapitalmarktrecht der Westfälischen WilhelmsUniversität Münster.

1 Napoleoni/Segre, Della finanza islamica proposte e idée per l'Occidente in crisi, L'Osservatore Romano v. 4.3.2009, S. 3, in diesen Chor stimmten aber auch weitaus namenhaftere Ökonomen ein, vgl. die Nachw. bei Bälz, ZVgIRWiss 109 (2010), 272 mit Fn. 1 f. sowie weit. Nachw. bei Momen, RIW 2010, 367, 368 in Fn. 4. 
teten Anlageprodukten. Exemplarisch sei auf die acht Kirchenbanken ${ }^{2}$ in Deutschland oder die EthikBank ${ }^{3}$ verwiesen. Auf der ökologischen Welle reitet etwa die umstrittene Prokon-Finanzgruppe, die jüngst wieder marktschreierisch warb: Prokon Genussrechte: rentabel, flexibel und einfach - aktuell $8 \%$ Zinsen p.a. mit erneuerbaren Energien. ${ }^{4}$ Ein Schelm, der Böses dabei denkt. Im Internet gibt es ganze Foren von geschädigten Anlegern von Vorgängerfonds. ${ }^{5}$

Dieser Überblick soll genügen, um das Spektrum und die Farbpalette zu umreißen, die die Pinselstriche für diesen Aufsatz liefern sollen.

Es soll in erster Linie um die Frage gehen, wie die religiösen, ethischen oder ökologischen Wertvorstellungen in den jeweiligen Finanzierungsvertrag transportiert werden, wie die Einhaltung der Wertvorstellungen überwacht wird und wer das Risiko trägt, wenn sich die Anlage letztlich nicht als ethisch erweist bzw. nicht den religiösen Vorgaben genügt. Dabei sollen Gemeinsamkeiten und Unterschiede zwischen dem Islamic Banking einerseits und dem sogenannten Ethikbanking andererseits aufgezeigt werden. Vorab sind aber die Begrifflichkeiten klarzustellen und die für die Untersuchung erforderlichen Rechtsfragen aus einer kurzen Bestandsaufnahme herauszudestillieren.

\section{Begrifflichkeiten und Bestandsaufnahme}

Islamic Banking oder Islamic Finance werden zumeist synonym gebraucht. Teilweise wird auch von shariakonformen Bankgeschäften gesprochen.

Ein gemeinsames Kennzeichen besteht darin, dass eine Bank oder ein Finanzdienstleister Finanzprodukte anbietet, die den Vorgaben des islamischen Rechts (der Sharia) genügen, ohne dass dieses zugleich zum anwendbaren Recht erklärt wird. Vielmehr kennzeichnet shariakonformes Banking, dass die Finanzierungsverträge einer staatlichen Rechtsordnung unterstellt werden, aber durch Ausgestaltung des

2 Auf katholischer Seite ist in Deutschland vor allem die Pax-Bank in Köln sowie die Liga-Bank in Regensburg, die Bank für Kirche und Caritas in Paderborn, die DKM Darlehenskasse Münster sowie die BANK IM BISTUM ESSEN auf evangelischer die Ev. Darlehensgenossenschaft in Kiel, die Ev. Kreditgenossenschaft in Kassel sowie die KD-Bank - Bank für Kirche und Diakonie in Dortmund mit ihrer Tochtergesellschaft der Landeskirchlichen Kredit-Genossenschaft in Sachsen zu nennen. Zur Kritik an der Anlagepolitik einiger dieser Institute etwa http://www.spiegel.de/spiegel/0,1518,717173,00.html (zuletzt aufgerufen am 29.3.2011).

$3 \mathrm{Vgl}$. http://www.ethikbank.de/die-ethikbank.html (zuletzt abgerufen am 14.3.2011). Hierbei handelt es sich um eine (rechtlich unselbständige) Zweigniederlassung der Volksbank Eisenberg eG.

4 Weiter liest man „wir haben Lust auf eine lebenswerte Zukunft“, garniert wird das Ganze mit einem Fuchs auf grüner Wiese vor einem Windkraftrad, der unschwer an den Fuchs der Bausparkasse Schwäbisch Hall erinnert und den Interessenten in vermeintlicher Sicherheit wiegen soll, vgl. Postwurfsendung vom Januar 2011; zu untersagten früheren Werbekampagnen vgl. auch LG Itzehoe v. 15.3.2011 (5 O $66 / 10)$ - juris.

5 Vgl. http://www.photovoltaikforum.com/finanzen-steuern-f9/prokon--t10219.html; sowie zu den aktuellen Genussrechten ferner http://www.test.de/themen/geldanlage-banken/meldung/Prokon-Genussrechte-Windige-Werbung-1852031-2852031/\# (beide zuletzt aufgerufen am 29.3.2011). 
Vertrages und der Anlagepolitik den Vorgaben des islamischen Rechts Genüge getan wird. ${ }^{6}$ Dabei versucht man insbesondere dem Zinsverbot (riba-Verbot) sowie dem Spekulationsverbot (Gharar) und dem Verbot des Glücksspiels (Maisir) zu genügen. Die Anbieter von islamischen Finanzprodukten sind vielfältig. Es lassen sich vor allem drei Gruppen aus einer bunten Schar herausziselieren. Zum einen gibt es sogenannte islamische Vollbanken, die ausschließlich islamkonforme Bankgeschäfte betreiben und ihre gesamte Geschäftspolitik an den Vorgaben der Sharia ausrichten. Daneben existieren sog. islamische Teilbanken, die sowohl konventionelle, also zinsgesteuerte Bankgeschäfte wie auch islamische Bankgeschäfte anbieten ( „Islamic Windows “). ${ }^{7}$ Drittens sind schließlich Anbieter einzelner islamischer Finanzprodukte zu beobachten, die nicht zugleich über eine staatliche Banklizenz verfügen. ${ }^{8}$ Hinzu tritt eine weitreichende Liste von Ge- und Verboten, etwa dem Verbot in Unternehmen $\mathrm{zu}$ investieren, die Schweinefleisch oder Alkohol produzieren. Ein gemeinsames Kennzeichen aller Anbieter besteht darin, dass ein meist beim Unternehmen angesiedeltes, aber von dem operativen Geschäft getrenntes Gremium darüber wacht, dass die religiösen Vorgaben auch wirklich eingehalten werden. Diesen sog. Sharia Boards kommt also eine Zertifizierungsfunktion zu. Bildlich kann man von einem durch das Unternehmen selbst eingerichteten und finanzierten religiösem TÜV sprechen. Hintergrund ist das Fehlen einer zentralen Instanz im Islam einerseits und klarer Vorgaben für Finanzgeschäfte andererseits. Die Mitglieder in diesen Boards sind im Gegensatz zu sogenannten Shariaberatern nicht unmittelbar im Unternehmen beschäftigt, sondern unabhängige, ökonomisch vorgebildete islamische Rechtsgelehrte, die am ehesten einem Aufsichtsrat vergleichbar sind, ohne freilich die gesamte Geschäftspolitik des Unternehmens zu kontrollieren. Sharia Boards können mit anderen Worten neben einem konventionellen Aufsichtsrat nach deutschem Verständnis existieren. ${ }^{9}$

Ähnlich strukturiert ist das Ethikbanking. Sehr vereinfacht ausgedrückt tritt an die Stelle der religiösen Vorgaben des Islam eine Weltanschauung oder ein diffuses Gemisch aus ökologischen, ethischen und sonstigen Vorgaben, etwa dem Gebot zum nachhaltigen Wirtschaften. Pars pro Toto steht die EthikBank, ${ }^{10}$ die ihre Unternehmensphilosophie auf ihrer Homepage wie folgt beschreibt: „Basis aller Geschäfte der EthikBank ist ihre sozialökologische Anlagepolitik - ein Mix aus Tabu- und Posi-

6 Vgl. nur Casper, ZBB 2010, 345, 346; Grieser, WM 2009, 586.

7 Vgl. näher zur globalen Marktstruktur des Islamic Banking etwa Gassner/Wackerbeck, Islamic Finance, 2. Aufl. 2010, S. 49 ff.; Saggau, in: Ebert/Thiessen (Hrsg.), Das islamkonforme Finanzgeschäft, S. 120 ff. Vgl. zum Potenzial islamischer Bankprodukte in Deutschland, Alexander/Blume/Braune, Das islamkonforme Finanzgeschäft, 2010, S. 82 ff.

8 Zur aufsichtsrechtlichen Problematik vgl. Casper, ZBB 2010, 345 ff.

9 Zur Verortung von Sharia Boards im westlichen Verständnis von Corporate Governance vgl. etwa Casper, FS Hopt, 2010, S. 457, 465 ff.

10 Vgl. oben Fn. 3. 
tivkriterien. So investiert die EthikBank das Geld ihrer Kunden nicht in Rüstungsgeschäfte, Atomkraft oder in Unternehmen, die Kinderarbeit zulassen. Die Unternehmen, die Geld von der EthikBank bekommen, müssen sich aktiv für eine nachhaltige Wirtschaftsweise engagieren."11 Dies wird sodann näher präzisiert. Wie bei islamischen Banken wird die Negativliste weiter ausgebaut und z.B. auf die Finanzierung der Herstellung von gentechnisch verändertem Saatgut und Pflanzen oder kosmetischer Produkte, die auf Tierversuchen fußen, ausgedehnt. Auch der „Ankauf von Staatsanleihen von den unter Nachhaltigkeitsaspekten unterdurchschnittlichen OECD-Staaten“ oder „von Ländern, die Menschenrechte verletzten“ wird verworfen. Ebenso sind Geschäfte mit radikalen Parteien ausgeschlossen. Die Kumulation von unbestimmten Begriffen schreit geradezu nach einer Zertifizierung. Ein dem Sharia Board vergleichbarer Ethikrat ist aber nicht durchweg zu beobachten. So arbeitet die EthikBank etwa mit einer internen Eigenkontrolle und einer weiterreichenden Transparenz ihrer Anlage- und Finanzierungspolitik („gläserne Bank“) und orientiert sich bei der Umsetzung ihrer Positiv- und Negativlisten an Vorgaben spezieller Unternehmensberatungsgesellschaften wie der ,imug“ in Deutschland oder dem Ethical Information and Research Service (EIRIS) in London. Spezielle Aufsichtsgremien sind jedoch auch in diesem Sektor keine Unbekannte. So hat das Bundesamt für Kreditwesen als Vorläufer der BAFin bereits 1989 im Zusammenhang mit sog. Ethik- und Ökofonds hervorgehoben, dass mit religiösen oder sonstigen Vertretern besetzte Anlageausschüsse nicht maßgeblich in die Geschäftspolitik eingreifen dürfen und die Kriterien für die Anlagepolitik transparent zu machen sind. ${ }^{12}$

Die Kirchenbanken lassen sich in Deutschland nicht über einen Leisten schlagen. Es gibt solche, die sich einfach als spezieller Dienstleister für kirchliche Institutionen verstehen, aber sich keine spezifischen Beschränkungen ihrer Geschäftspolitik auferlegen. ${ }^{13}$ Insbesondere unterscheidet sie von islamischen Banken, dass sie nicht den Anspruch erheben, mit ihrer Geschäftspolitik bzw. Anlagepolitik religiösem Recht, etwa dem kanonischen Recht, zu genügen. Einige kirchliche Banken lassen sich dagegen eher im Bereich des Ethikbanking verorten. So hat die Pax-Bank beispielsweise einen Ethikkodex aufgestellt. Darin finden sich aber deutlich weniger konkrete Vor-

11 Vgl. http://www.ethikbank.de/die-ethikbank.html (zuletzt aufgerufen am 29.3.2011) - Hervorhebung im Original.

12 Schreiben des BAKred vom 8. Juni 1989 (Az. V 4/51) zu den „Anforderungen an die Umschreibung der Anlagegrundsätze, Funktion von Anlageausschüssen“ abgedruckt in Beckmann/Scholtz, Investment, Bd. 2, Loseblattsammlung Stand 4/2008, Kz. 438 Nr. 46 sowie ebenfalls verfügbar unter http:// beck-online.beck.de/?vpath=bibdata\%2Fges\%2FKWG_10_44 \%2Fcont\%2FKWG_10_44.htm (zuletzt aufgerufen am 29.3.2011).

13 So beschreibt z.B. die Ev. Darlehensgenossenschaft Kiel ihr Selbstverständnis unter der Überschrift „Kompetenz in allen Finanzfragen“ wie folgt: „So vereinen wir die Stärken eines modernen Kreditinstitutes mit dem umfassenden Know-how des in der Tradition ihrer Kunden verwurzelten Spezialdienstleisters für Kirchen, Diakonie, Caritas, Freie Wohlfahrtspflege und diesen Institutionen nahestehenden Privatkunden.“, vgl. http://www.edg-kiel.de/mensch_deine_bank.html (zuletzt aufgerufen am 30.3.2011). 
gaben als etwa in den Grundsätzen für die Anlagepolitik der EthikBank. ${ }^{14}$ Vor allem wird in dem Kodex der Pax-Bank betont, dass die dort aufgestellten „ethische[n] Verpflichtungen, die das Unternehmen und seine Mitarbeiter freiwillig aus eigener Überzeugung und unabhängig von Rechtspflichten auf sich nehmen “, keinerlei rechtliche Verpflichtungen auslösen sollen. ${ }^{15}$ Auch enthält der Kodex vor allem Allgemeinplätze und sein Konkretisierungsgrad erinnert mehr an das allwöchentliche Wort zum Sonntag, denn an im Geschäftsalltag umsetzbare konkrete Handlungsanweisungen. Ein Ad-hoc-Ethikbeirat hatte nur einmalig beratende Aufgabe bei Aufstellung des Kodexes, ihm kommt aber keine laufende Überwachungs- oder Zertifizierungsfunktion wie einem Sharia Board zu. Insgesamt lässt sich also festhalten, dass die sog. Kirchenbanken in Deutschland nur bedingt mit ethisch ausgerichteten Banken vergleichbar sind, meist handelt es sich bei ihnen nur um eine Spezialbank für kirchliche Einrichtungen, die jedoch im Wesentlichen allgemeine Bankgeschäfte betreiben.

Abzugrenzen ist der Begriff Corporate Social Responsibility (CSR). Während religiös oder ethisch motivierte Bankgeschäfte bestimmten Wertvorstellungen genügen müssen, handelt CSR von der Frage, inwieweit der Vorstand einer Kapitalgesellschaft das ihm anvertraute Vermögen des Unternehmens auch für soziale Projekte verwenden und ob er bei seinen unternehmerischen Entscheidungen soziale und ethische Belange berücksichtigen darf, auch wenn hierdurch der zu erwartende Gewinn geschmälert wird. ${ }^{16}$ Anders als bei religiös oder ethisch ausgerichteten Banken ist diese Tätigkeit stets nur ein Nebenzweck, teilweise verbunden mit der Erwartung, durch das soziale Engagement langfristig den Gewinn des Unternehmens oder zumindest seine Reputation zu steigern. ${ }^{17}$

Demgegenüber weist der Begriff Socially Responsible Investment (SRI) deutlich mehr Gemeinsamkeiten mit dem hiesigen Thema auf. Unter diesem Stichwort werden die rechtlichen Rahmenbedingungen bezeichnet, mit denen Investment- oder Pensionsfonds bei ihrer Anlagepolitik neben unmittelbar finanziellen Faktoren auch ethische, soziale oder ökologische Kriterien mit einfließen lassen. ${ }^{18}$

14 Deutlich präziser sind der Nachhaltigkeitsfilter der KD-Bank, vgl. http://www.kd-bank.de/etc/medialib/i500m0320/01_KD-Bank_allgemein/pdf.Par.0012.File.tmp/Nachhaltigkeitsfilter_web.pdf sowie die Anlagegrundsätze der Bank im Bistum Essen, vgl. http://www.bibessen.de/nachhaltigkeit/nachhaltige_geschaeftspolitik/anlagegrundsaetze.html (beide zuletzt aufgerufen am 30.3.2011).

15 Ethikkodex der Pax-Bank, 20005, S. 27 - verfügbar unter http://www.paxbank.de/fileadmin/ user_upload/pdf/sonstige/ethik-kodex.pdf (zuletzt aufgerufen am 30.3.2011).

16 Vgl. dazu neuerdings ausführlich Müller-Michaels/Ringel, AG 2011, 101, 103 ff. mit ausführlicher Darstellung des Meinungsstandes.

17 Zu CSR im Kontext des Islamic Banking vgl. Nathan/Pierce, in: Mallin (Hrsg.), Corporate Social Responsibility: A Case Study Approach, 2009, 258-273.

18 Ausführlich zum Ganzen etwa Scharau, Socially Responsible Investment, 2009, S. 7 ff. und passim sowie kürzlich Preu/Richardson, German Law Journal 2011, 865 ff., abrufbar unter http://www.germanlawjournal.com/index.php?pageID=11\&artID=1345 (zuletzt aufgerufen am: 10.5.2011). 
Dieser Beitrag will jedoch im Folgenden den Finanzierungsvertrag in den Vordergrund stellen und dabei untersuchen, wie sich Störungen der religiösen und weltanschaulichen Vorstellung auswirken. Als einheitlicher Begriff wird dafür die Konformität mit außerrechtlichen Vorgaben gewählt.

\section{B. Destillation von Rechtsfragen}

Rückt man den Finanzierungsvertrag in den Vordergrund, der zwar einer staatlichen Rechtsordnung unterliegt, ${ }^{19}$ aber vor allem religiösen oder selbst gesetzten ethischen Zielen genügen will, lassen sich einige bisher wenig beleuchtete Rechtsfragen herausdestillieren. Diesem Ansatz könnte man auf den ersten Blick entgegenhalten, dass zumindest in solchen Rechtsordnungen, die von Vertragsfreiheit gekennzeichnet sind, ein derartiger Parteiwille regelmäßig keine Schwierigkeiten bereiten sollte. Daran ist richtig, dass die Privatautonomie auch darin zum Ausdruck kommt, dass ein Anleger solche Anlageprodukte wählen kann, die mit seinen religiösen oder sonstigen weltanschaulichen Zielsetzungen im Einklang stehen. Andererseits muss sich auch eine weltanschaulich neutrale Rechtsordnung, die vom Grundsatz der Privatautonomie geprägt ist, zu der Frage verhalten, wie sich die Verfehlung solcher gewissermaßen außerrechtlichen Vorgaben auf die vertragliche Risikoverteilung zwischen Anleger und Finanzdienstleister auswirkt.

Deshalb wird in einem ersten Schritt zu überlegen sein, ob der gesamte Vertrag unwirksam oder aber bei Dauerschuldverhältnissen zumindest beendet werden kann. In diesem Zusammenhang wird danach zu fragen sein, inwieweit die Kompatibilität des Finanzprodukts mit den ethischen oder religiösen Vorgaben zur Geschäftsgrundlage des Vertrages werden kann (sub C.). Dabei wird es bereits maßgeblich auf die Frage ankommen, wie das Risiko für eine Fehleinschätzung der außerrechtlichen Vorgaben zu verteilen ist. Die Extrempositionen könnten auf der einen Seite lauten, dass die Nichterfüllung der ethischen oder religiösen Zielvorstellungen ein unerhebliches Affektionsinteresse der jeweiligen Vertragspartei sei, weshalb sich der Anleger im Vorfeld gefälligst selber darum zu kümmern habe, ob die Anlage seinen Vorstellungen genüge. Auf der anderen Seite stände dann die These, dass der Emittent eines Finanzproduktes stets die Konformität mit der Sharia bzw. die Übereinstimmung mit den beworbenen ethischen Zielen garantiere. ${ }^{20}$

Verneint man eine Störung der Geschäftsgrundlage bei Verfehlung der außerrechtlichen Vorgaben, ist die Frage aufzuwerfen, ob das religiöse Recht oder die ethischen

19 Im Folgenden soll es nicht abermals um die bereits häufiger diskutierte Frage gehen, ob nach den Grundsätzen des deutschen Internationalen Privatrechts in einem Vertrag auf eine nichtstaatliche Rechtsordnung wie das islamische Recht verwiesen werden kann, vgl. dazu ausführlich - ablehnend - Bälz, IPrax 2005, 44, 45; ders., in: Kronke (Hrsg.), Islamisches und Arabisches Recht als Problem der Rechtsanwendung, 2001, 63, 68; Junius, Chicago Journal of International Law, 2006/2007, 537, $546 \mathrm{ff}$.

20 Zum Diskussionstand vgl. einstweilen nur Bälz, BKR 2002, 447, 451. 
Vorgaben zumindest im Wege der Vertragsauslegung - etwa im Zusammenhang mit der teleologischen Auslegung - zu berücksichtigen sind (sub D.). Im konkretisierenden Zugriff geht es also darum, ob der Vertrag im Lichte der einschlägigen Vorgaben der Sharia auszulegen ist, obwohl das Vertragsstatut säkulares, nationales Recht ist. ${ }^{21}$ Soweit man dies annehme würde, stellt sich die Folgefrage, ob das bei Rechtsstreitigkeiten berufene nationale Gericht insoweit in der Lage ist, letztverbindlich zu bestimmen, was überhaupt die Vorgaben des religiösen Rechts sind bzw. wie Nachhaltigkeit bei einem Nachhaltigkeitsfonds letztlich zu definieren ist oder ob insoweit ein Rechtsgutachten einzuholen ist.

Schließlich ist den Rechtsfolgen - insbesondere möglichen Haftungsansprüchen - bei Verfehlung der außerrechtlichen Vorgaben nachzugehen (sub E.). Insoweit sind zwei Fragen auf das Trapez gebracht. Zum einen ist zu klären, ob der Anbieter für eine ursprüngliche Verfehlung der außerrechtlichen Ziele haftet, insbesondere wenn er hierauf in einem Prospekt hingewiesen hat. Zum anderen ist zu analysieren, was bei der Änderung der Einschätzung nach Abschluss des Vertrages passiert. Konkretisierend ist also den Folgen einer Änderung der Einschätzung der Shariakonformität oder der Umweltfreundlichkeit etc. nachzuspüren, die sich erst während der Vertragslaufzeit ergeben hat. Im Zusammenhang mit Islamic Finance hat insoweit das Wort von der Shariafalle die Runde gemacht. ${ }^{22}$ Soweit man den Emittenten grundsätzlich in der Pflicht sieht, bleibt zu klären, ob er dieses Risiko auf den Anleger abwälzen kann.

\section{Die Kompatibilität mit außerrechtlichen Vorgaben als Vertragsgrundlage?}

\section{Erfahrungen aus der englischen Gerichtspraxis}

Im Folgenden wird also unterstellt, dass der Vertrag nicht unmittelbar auf eine nichtstaatliche Rechtsordnung als anwendbares Recht verweisen will, sich wohl aber deren Vorgaben - ganz oder zumindest in Teilen - unterwerfen will. Standardbeispiel hierfür sind islamische Finanzierungsverträge. Paradigmatisch ist die Entscheidung des Londoner Court of Appeal in Sachen Shamil Bank of Bahrain vs. Beximco aus dem Jahre 2004. ${ }^{23}$ Die Parteien hatten einen Finanzierungsvertrag auf Basis einer sog. Murabaha geschlossen. Dabei handelt es sich sozusagen um die islamische Variante des Abzahlungskaufs. Im unproblematischen Prototyp kauft die Bank das von ihrem Kunden zu finanzierende Wirtschaftsgut zunächst selbst zum Barpreis an, um es sodann mit einem Aufschlag an den Kunden weiterzuverkaufen. Dabei wird der

21 Bälz, ZVglRWiss 109 (2010), 272, 286.

22 Überblick über die Diskussion bei Casper in Jansen/Oestmann (Hrsg.), Gewohnheit, Gebot, Gesetz Zur Entstehung von Normen in Geschichte und Gegenwart, im Erscheinen 2011, S. 301, $323 \mathrm{ff}$.

23 Urt. v. 28.1.2004, (2004) All ER (D) 280 (Jan.). Auf einer ähnlichen Linie liegt das Urteil in Sachen Islamic Investment Company of the Gulf vs. Symphony Gems; zu diesem Urteil ausführlich vgl. Bälz, Islamic Financing Transactions in European Courts, in: Al-Harmaneh/Thielmann, Islam and Muslims in Germany, 2008, S. 569, $571 \mathrm{ff}$. 
höhere Kaufpreis aus dem Weiterkauf in der Regel gestundet und sodann in Raten vom Endkäufer und Bankkunden gezahlt. ${ }^{24}$ In dem konkreten Fall war der eigentliche Kaufgegenstand in den Hintergrund und die Finanzierungsfunktion in den Vordergrund gerückt. Als Beximco nunmehr mit Zahlung der Raten in Verzug geraten war und auf Zahlung gerichtlich in Anspruch genommen wurde, verteidigte sich das Unternehmen flugs damit, dass der Vertrag bei Lichte betrachtet gegen das koranische Zinsverbot verstoßen würde. Der Vertrag über die Murabaha sei folglich unwirksam, weshalb auch keine Rückzahlungspflicht bestehe. Sie berief sich dabei auf folgende Klausel in dem Vertrag: „Subject to the Principles of Glorious Sharia, this Agreement shall be governed by and construed in accordance with the laws of England." Zwar unterliege der Vertrag englischem Recht, aber durch den gleichzeitigen Verweis auf die Sharia müsse der Verstoß gegen das Zinsverbot auf die Wirksamkeit des Vertrages durchschlagen. Dem ist der Court of Appeal mit überzeugenden Gründen entgegen getreten. Das Gericht sieht sich nicht an diese „hybride Rechtswahl“ 25 gebunden. Zur Begründung wird vor allem darauf verwiesen, dass die Grundsätze der Scharia nicht hinreichend bestimmt seien. Dabei sei der (hypothetische) Wille der Parteien so auszulegen, dass er der ausdrücklich gewollten und auch wirksamen Wahl englischen Rechts als Vertragsstatut nicht zuwiderläuft. Ausgehend hiervon sei die von den Parteien als „Rechtswahl“ bezeichnete Klausel über die Shariakonformität lediglich so zu verstehen, dass die Bank ihr Geschäftsgebaren an den religiösen Prinzipien des Islam ausrichte. Im Übrigen könne das Gericht den Aussagegehalt islamrechtlicher Grundsätze mangels Sachkunde jedoch nicht prüfen und es könne auch kaum dem Willen der Parteien entsprechen, ein (säkulares) englisches Gericht mit der Auslegung des islamischen Rechts zu betrauen. Letzeres stimmt auf den ersten Blick zwar bedenklich, denn dieses Problem stellt sich häufig, insbesondere bei dem Verweis auf ausländisches Recht und lässt sich mit der Einholung von Gutachten lösen. Der Unterschied zwischen dem Verweis auf eine fremde Rechtsordnung und auf das islamische Recht liegt freilich darin, dass es „das“ islamische Recht gar nicht gibt. Vielmehr ist die Interpretation, wie das islamische Recht auszulegen ist und welche konkreten Ver- und Gebote es aufstellt, abhängig davon, welcher der verschiedenen Rechtsschulen oder -traditionen man folgt. ${ }^{26}$ Einige sprechen sogar von einer normativen Ambiguitätstoleranz des Islams. ${ }^{27}$

Richtig an der Entscheidung ist auch, dass der Unwirksamkeitsgrund einer anderen Rechtsordnung wie des islamischen Rechts, nicht einfach durch einen schlichten

24 Vgl. näher zur Murabaha etwa Gassner/Wackerbeck (Fn. 7), S. 52 ff.; Vogel/Hayes, Islamic Law and Finance, 2006, S. $140 \mathrm{ff}$.

25 Ausdruck nach Bälz, ZVglRWiss 109 (2010), 272, 285.

26 Allein im sunnitischen Islam gibt es mindestens vier Rechtschulen mit teilweise sehr unterschiedlichen Traditionen, vgl. näher zu ihnen etwa Hallaq, An Introduction to Islamic Law, 2009, S. 38 ff.

27 Bauer, in: Jansen/Oestmann, Gewohnheit, Gebot, Gesetz - Zur Entstehung von Normen in Geschichte und Gegenwart, im Erscheinen 2011. 
Hinweis auf die eigentlich gewählte Rechtsordnung durchschlagen kann. Hierfür bedürfte es einer ausdrücklichen, durch die gewählte Rechtsordnung legitimierten Anknüpfung. Denkbar wäre freilich, dass die Parteien im Rahmen der Vertragsfreiheit einen entsprechenden Unwirksamkeitsgrund in den Vertrag schreiben.

$\mathrm{Zu}$ einem gegenläufigen Ergebnis scheint aber der English High Court in seinem vorläufigen Urteil vom 11.12.2009 in Sachen Blom Development Bank (Blom) vs. The Investment Dar Company (TID) zu gelangen. ${ }^{28}$ Die Klägerin, eine libanesische Bank, hatte bei der Beklagten (der TID), einem kuwaitischen Finanzdienstleister im Bereich des Islamic Finance, 10 Million US-Dollar angelegt. Dafür hatten die Parteien eine sogenannte Wakala-Konstruktion gewählt, eine Art Vermögensverwaltungsvertrag, nach dem die Beklagte das Geld ausschließlich shariakonform anlegen sollte. Bei einer Wakala-Konstruktion ${ }^{29}$ wird nach islamischem Rechtsverständnis und gängiger Finanzpraxis ${ }^{30}$ ein Vermögenspool aus den Vermögensgegenständen einer Person oder mehrerer Anleger gebildet. Anders als bei den typischen Wakala-Konstruktionen, bei denen der Gewinn ungewiss ist und der Anleger auch Verluste tragen muss, wurde der klagenden Investorin nicht nur die Rückzahlung des angelegten Kapitals, sondern auch eine Mindestrendite versprochen. Ein Verstoß gegen das ribaVerbot war also mit Händen zu greifen. Gleichwohl hatte der Sharia Board der Beklagten die Vertragskonstruktion als islamkonform abgesegnet. Die Beklagte, eine kuwaitische Aktiengesellschaft, war obendrein bereits nach ihrer Satzung verpflichtet, ausschließlich shariakonforme Finanztransaktionen abzuschließen und keine ri$b a$-Geschäfte zu tätigen. ${ }^{31} \mathrm{Im}$ Wege der Rechtswahl wurde englisches Recht für anwendbar erklärt, eine „hybride Rechtswahl“ wie in der Entscheidung Shamil Bank of Bahrain v. Beximco fand sich nicht.

Der High Court verurteilte die Beklagte, die jegliche Zahlungen mit Hinweis auf den Verstoß gegen das riba-Verbot abgelehnt hatte, in seiner vorläufigen Entscheidung jedoch nur zur Rückzahlung des angelegten Kapitals, die Zahlung von Erträgen wurde abgelehnt. Zur Begründung wurde darauf verwiesen, dass der Vertrag voraussichtlich wegen eines Verstoßes gegen die Grundsätze der ultra-vires-Lehre ${ }^{32}$ un-

28 [2009] EWHC 3545 (Ch); verfügbar unter Westlaw als 2009 WL 5386898. Das Verfahren ist noch nicht abgeschlossen.

29 Zur Wakala vgl. etwa Ayub, Understanding Islamic Finance, 2009, S. 347 ff.

30 In der Entscheidung des High Courts (Fn. 28) wird im Zusammenhang mit der Erläuterung dieser Vertragskonstruktion auf das Merkblatt der britischen HM Revenue \& Customs - VATFIN8500 Islamic Products: Agency (Wakala) verwiesen, abrufbar unter: http://www.hmrc.gov.uk/manuals/vatfinmanual/vatfin8500.htm (zuletzt aufgerufen am 20.5.2011).

31 Art. 5 des Memorandum of Association von TID lautet (in der engl. Übersetzung): „The objectives for which the company is established shall be Sharia compliant. None of the objectives shall be construed and interpreted as permitting the company to practice directly or indirectly any usury or non-Sharia compliant activities." - zitiert nach der Entscheidung des High Courts (Fn. 28).

32 Vgl. zur im angelsächsischen Recht verbreiteten ultra-vires-Lehre Mayson/French/Rayon, Company Law, 2007, S. 97 f.; Cahn/Donald, Comparative Company Law, 2010, S. 313 f. 
wirksam sei, ${ }^{33}$ da die gewählte Vertragskonstruktion im Ergebnis zu einer festen Verzinsung führe und somit gegen das riba-Verbot verstoße. Damit wird zwar nicht die Sharia zum anwendbaren Recht erklärt. Jedoch wird über den Rückgriff auf die ultra-vires-Lehre die fehlende Shariakonformität zum Unwirksamkeitsgrund erhoben und das Urteil des Sharia Boards in Frage gestellt.

Prima vista wird der Eindruck erweckt, dass sich das Gericht im Wege einer summarischen Prüfung anmaßt, das Urteil der islamischen Rechtsgelehrten in Frage zu stellen, ohne selbst ein Rechtsgutachten erstellen zu lassen. Gleichwohl erscheint die gewählte Konstruktion als offensichtlich mit dem islamischen Zinsverbot unvereinbar. $^{34}$

\section{Fehlende Shariakonformität als Vertragsgrundlage?}

Im deutschen Recht wird die ultra-vires-Lehre bekanntlich nicht anerkannt. ${ }^{35}$ Mit der Argumentation des High Courts läge es aus deutscher Perspektive aber nahe, die fehlende Konformität mit der Sharia zur Geschäftsgrundlage zu erklären und den Vertrag über $\$ 313$ Abs. 3 BGB zu Fall zu bringen. Die Konstellationen in den beiden englischen Entscheidungen scheinen sich auf den ersten Blick zwangslos unter $\$ 313$ Abs. 2 BGB subsumieren zu lassen, so denn die Vereinbarkeit mit außerrechtlichen Vorgaben wirklich eine Geschäftsgrundlage des Vertrages bildet.

\section{1. Überblick zur Diskussion um die Geschäftsgrundlage im deutschen Recht}

Nach ständiger Rechtsprechung wird die Geschäftsgrundlage eines Vertrages gebildet durch „die bei Vertragsschluss bestehenden gemeinsamen Vorstellungen beider Parteien oder die dem Geschäftsgegner erkennbaren und von ihm nicht beanstandeten Vorstellungen der einen Vertragspartei vom Vorhandensein oder dem künftigen Eintritt gewisser Umstände, sofern der Geschäftswille der Parteien auf dieser Vorstellung aufbaut““. ${ }^{36}$ Dieses subjektive Verständnis der Geschäftsgrundlage liegt insbesondere $\mathbb{3 1 3}$ Abs. 2 BGB zugrunde. Dagegen klingt in $\$ 313$ Abs. 1 BGB eher ein objektives Verständnis der Geschäftsgrundlage an, die also nicht an die Vorstellung

33 Wörtlich heißt es in den Urteilsgründen (vgl. Fn. 28): „The agreement had been entered into beyond the corporate powers of TID and was therefore void”. Das Gericht hält damit die Beschränkung der Vertretungsbefugnis durch die Satzung der Beklagten, einer kuwaitischen Aktiengesellschaft, für das englische Vertragsstatut für maßgeblich, ohne dies in seiner vorläufigen Entscheidung näher kollisionsrechtlich zu begründen. Die Beschränkung der Vertretungsmacht wird also nicht zur selbständig anzuknüpfenden Vorfrage erhoben.

34 Diese Annahme kommt auch im richterlichen Votum (Fn. 28) bei der Beurteilung der streitgegenständlichen Wakala-Konstruktion - wenn auch etwas zurückhaltend - zum Ausdruck, wenn es heißt: "one finds [...] in this wakala contract, a device to enable what would at least to some eyes appear to be the payment of interest under another guise, that is at least an indirect practice of a non-Sharia compliant activity".

35 Vgl. nur K. Schmidt, Gesellschaftsrecht, 4. Aufl., 2002, $\$ 8$ V, S. 214 ff.; Zöllner/Noack, in: Baumbach/ Hueck, GmbHG, $\mathbb{3} 35$ Rn. 80.

36 So zuletzt BGH NJW 2010, 1663; vgl. auch die umfangreichen Nachweise bei MüKo-BGB/Roth, \$313 Rn. 47, Bamberger/Roth/Unberath, $\$ 313$ Rn 4. 
der Parteien anknüpft, sondern an die objektiv nötigen Voraussetzungen, damit sich der Wille der Parteien noch sinnvoll verwirklichen kann. ${ }^{37}$ Das tradierte Standardbespiel bildet bekanntlich die schwerwiegende Äquivalenzstörung. ${ }^{38}$ Dieser objektive Ansatz wurde in der Vergangenheit bereits von der Literatur propagiert, wohingegen die Rechtsprechung solche Umstände eher durch eine großzügige Interpretation der subjektiven Geschäftsgrundlage erfassen will, die nicht voraussetze, dass die Parteien über die relevanten Umstände gesprochen hätten. ${ }^{39}$

Dieser Beitrag nimmt nicht für sich in Anspruch, eine qualifizierte Stellungnahme zu den Tiefen und Untiefen der Diskussion um die Geschäftsgrundlage abzugeben. Allerdings lässt sich für die folgenden Überlegungen angesichts der Systematik des \$313 BGB die Arbeitshypothese aufstellen, dass ein subjektives Verständnis der Geschäftsgrundlage, wie es in Abs. 2 anklingt, eine besondere Fallgruppe der grundsätzlich nach Abs. 1 objektiv zu ermittelnden Geschäftsgrundlage darstellt. Damit wird von $\$ 313$ BGB im Ergebnis sowohl die objektive, als auch die subjektive Geschäftsgrundlage erfasst. ${ }^{40}$

Nicht zur Geschäftsgrundlage gehören dagegen solche Umstände, die bereits Vertragsinhalt geworden sind. ${ }^{41}$ Vor diesem Hintergrund ist vorrangig eine Auslegung des Vertrages ( $\mathbb{S} 133,157 \mathrm{BGB}$ ) vorzunehmen, um festzustellen, ob nicht bereits hierdurch den Interessen der Parteien hinreichend Rechnung getragen werden kann. ${ }^{42}$

\section{Abgrenzung zur vertraglich übernommenen Risikoverteilung}

Eine allgemeine Antwort, ob die mangelnde Einhaltung außerrechtlicher Vorgaben eine Störung der Geschäftsgrundlage bildet, lässt sich angesichts der mannigfaltigen Ausgestaltung dieser Verträge kaum geben, sodass im Folgenden einige Leitlinien zu entwickeln sind, die bei der Beurteilung des Einzelfalls zugrunde gelegt werden können.

Vorrangig ist die Frage abzuschichten, ob die Verteilung des Risikos nicht bereits Vertragsbestandteil geworden ist und es somit einer der beiden Vertragsparteien zugewiesen werden kann. Eine pauschale Aussage, dass das Risiko der fehlenden Konformität stets bei der Bank oder dem Finanzdienstleister liege, kann nicht überzeugen.

37 Jauernig/Stadler, $\$ 313$ Rn. 4; vgl. auch BT-Drucks. 14/6040, S. 176, wonach Abs. 2 die subjektive Geschäftsgrundlage regele, Abs. 1 dagegen objektiver gefasst sei als die Formel der Rechtsprechung (wobei jedoch eine Änderung der Rechtsprechung hierdurch nicht veranlasst sei).

38 Grundlegend hierzu Larenz, Geschäftsgrundlage und Vertragserfüllung, 3. Aufl. 1963, S. 78 ff. sowie aus der aktuellen Kommentarliteratur etwa HK-BGB/Schulze, $\mathbb{S} 313$ Rn. 2.

39 Vgl. die Nachw. bei Palandt/Grüneberg, $\$ 313$ Rn. 4.

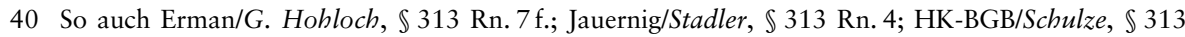
Rn. 2; im Ergeb. auch Palandt/Grüneberg, $\mathbb{S} 313$ Rn. 4.

41 So bereits Oertmann, Geschäftsgrundlage, 1923, S. $31 \mathrm{ff} ;$ vgl. auch PWW/Medicus/Stürner, $\mathbb{} 313$ Rn. 8 m. weit. Nachw.

42 Vgl. statt aller MüKo-BGB/Roth, $\mathbb{3 1 3}$ Rn. 130 m. weit. Nachw. 
Grundsätzlich handelt es sich bei der Verwirklichung von außerrechtlichen Vorgaben um ein unerhebliches Affektionsinteresse der Parteien. Andererseits würde es ebenfalls zu kurz greifen, das Risiko für eine Fehleinschätzung ausschließlich dem Anleger bzw. dem sonstigen Kunden des Finanzdienstleisters zuzuweisen. Denn schließlich ist es der Finanzdienstleister, der die Produkte als shariakonform, nachhaltig oder dergleichen mehr bewirbt und hiervon profitiert. Allerdings entspricht es dem Grundsatz der Privatautonomie, dass die Parteien derartige außerrechtliche Vorgaben zum Vertragsinhalt machen können. Das Risiko ist also zwischen den Parteien sachgerecht zu verteilen. Aufgabe der Bank ist es, zum einen nur solche Produkte als shariakonform anzubieten, bei denen nicht von vornherein offenkundig ist, dass sie mit den Grundsätzen der Sharia im Widerspruch stehen. Insoweit trifft sie eine Prüfungspflicht.

Im Übrigen obliegt es der Verantwortung der Bank, den Kunden entweder darauf hinzuweisen, dass sie in Zweifelsfällen keine Überprüfung der Konformität mit der Sharia vornimmt oder aber sie hat für eine solche Überprüfung Sorge zu tragen. Dabei muss die Bank durch eine anerkannte Einrichtung überprüfen lassen, ob ihr feilgebotenes Produkt den Anforderungen an die außerrechtlichen Vorgaben genügt. Dann ist sie im Gegenzug auch berechtigt, sich auf diese Einschätzung zu verlassen. Im Falle des Islamic Banking kann diese überprüfende Organisation ein von der Bank bestellter Sharia Board, ${ }^{43}$ ein sonstiger Gutachter oder ein Verband sein. Als Verbände kommen in Deutschland zum Beispiel der Zentralrat der Muslime in Betracht, auf internationaler Ebene die AAOIFI oder der IFSB. ${ }^{44}$ Ist der Anbieter islamischer Finanzprodukte diesen Anforderungen nachgekommen, kann er im Übrigen auf die Interpretationsvielfalt innerhalb des islamischen Rechts verweisen ${ }^{45}$ und sich darauf verlassen, dass ursprüngliche oder nachträgliche Fehleinschätzungen in die Risikosphäre des Anlegers fallen. Zwar wird man den Sharia Board in aller Regel nicht als Dritten im Sinne des $\$ 317$ BGB einordnen können, da er nur zertifiziert und nicht zugleich eine Leistungsbestimmung vornimmt. Allerdings lässt sich der dort enthaltene Rechtsgedanke, dass der Dritte - hier der Sharia Board - nach billigem Ermessen handelt, für die hier entwickelte These flankierend ins Feld führen.

Die mit Blick auf die Geschäftsgrundlage spannende Frage geht nun dahin, ob man diese soeben skizzierte Vertragsauslegung bei jedem islamkonformen Finanzierungsvertrag vornehmen kann, sodass stets eine entsprechende vertraglich vereinbarte $\mathrm{Ri}$ sikozuweisung vorliegt, die den Rückgriff auf $\$ 313$ BGB sperrt.

43 Zu dessen Aufgaben und Befugnissen sowie zu Corporate Governance Aspekten vgl. statt Vieler Casper, Festschrift Hopt, 2010, S. 457, 459, 466 ff.

44 AAOIFI = Accounting and Auditing Organization for Islamic Financial Institutions, Bahrain, vgl. http://www.aaoifi.com/overview.html und IFSB = Islamic Financial Services Board mit Sitz in Malaysia, vgl. http://www.ifsb.org.

45 Vgl. dazu schon oben mit Fn. 27. 
Dies wird man zumindest dann annehmen können, wenn der Finanzdienstleister seinen Pflichten nachgekommen ist, also eine Zertifizierung des Produkts hat vornehmen lassen und den Kunden auf die Möglichkeit von Fehleinschätzungen hingewiesen hat. In der bloßen Verwendung arabischer Terminologie für das gewählte Produkt wird man regelmäßig noch keine derartige vertragliche Abrede über die Risikoverteilung sehen können. Andererseits bildet diese Verwendung einer arabischen Terminologie regelmäßig noch keine hinreichende Grundlage dafür, dass beide Parteien die Konformität des Produkts mit der Sharia zur Grundlage des Vertrages erklären wollen. Schließt etwa eine Bank mit ihrem Kunden eine Mudaraba, auf die deutsches Recht zur Anwendung kommen soll, so wird damit nur zum Ausdruck gebracht, dass sich die Vertragsstruktur an einer Mudaraba orientiert, nicht aber auch, dass auch kein Verstoß gegen das riba-Verbot des Korans vorliegt. ${ }^{46}$ Eine derartige Inbezugnahme des islamischen Rechts muss zumindest im Vertrag anklingen oder sich aus den den Vertragsschluss begleitenden Umständen ergeben. Dies gilt umso mehr als die Parteien dann eine klare Vorstellung darüber entwickelt haben müssten, welcher Interpretation des riba-Verbots - nämlich einer eher formalen oder einer materiellen Sichtweise - ihr Vertrag folgen soll. So würde zum Beispiel die shafiitische Rechtschule in dem Beispielsfall Shamil Bank of Bahrain v. Beximico gar keinen Verstoß gegen die Vorgaben des islamischen Rechts erblicken. ${ }^{47}$

Damit lässt sich als Zwischenergebnis festhalten, dass für einen Rückgriff auf $\mathbb{} \$ 13$ BGB regelmäßig kein Raum ist. Entweder liegt kein hinreichender Hinweis darauf vor, dass die Konformität mit der Sharia wirklich Grundlage des Vertrages sein soll. Lässt sich diese Vorstellung der Parteien dem Vertrag bzw. den ihn begleitenden Umstände hingegen doch entnehmen, dann wird in aller Regel bereits ein entsprechender Vertragsinhalt vorliegen.

Dieses enge Verständnis der Geschäftsgrundlage muss erst recht für ethische oder ökologische Vorgaben gelten. Hat sich schon die mittelbare Inbezugnahme auf das islamische Recht als zu schillernd erwiesen, so gilt dies umso mehr für die noch viel diffusere Vorgabe, dass ein Investmentfonds beispielsweise nur in nachhaltig oder ökologisch wirtschaftende Unternehmen investieren darf. Will man die Einhaltung der Vorgaben einer bestimmten Organisation zu der Geschäftsgrundlage machen, bedarf es einer konkreten Bezeichnung von Grundsätzen, die einer Nachprüfung für Außenstehende zugänglich ist. Dann liegt jedoch wiederum ein Vertragsbestandteil

46 Bei der Mudaraba handelt es sich nach deutschem Verständnis um eine einer stillen Gesellschaft vergleichbaren Teilhaberschaft, Überblicke dazu etwa bei Ayub, Understanding Islamic Finance, 2007, S. 321 m. weit. Nachw; Casper, ZBB 2010, 345, 351 f.; Gassner/Wackerbeck, Islamic Finance, 2. Aufl. 2010, S. 79 f.; Zerwas/Demgensky, WM 2010, 692, 699; sowie die für die Bankpraxis wichtigen AAOIFI Standards on Mudaraba.

47 Zur shafiitischen Rechtsmeinung und allgemein zum umstrittenen Doppelkauf (bai ina) und zur sog. commodity murabaha (tawarruq) vgl. Warde, Islamic Finance in the Global Economy, 2. Aufl. 2010, S. $143 \mathrm{f}$. 
vor, so dass auch insoweit für einen Rückgriff auf die Grundsätze der Geschäftsgrundlage kein Raum ist.

\section{Mögliche Anwendungsfälle der Geschäftsgrundlage}

Als möglicher Anwendungsbereich für eine gestörte Geschäftsgrundlage sind damit allenfalls solche Fälle denkbar, in denen die Übereinstimmung der Anlage mit ethischen oder religiösen Vorgaben zwar von den Parteien angenommen und für wesentlich befunden wurde, ein entsprechender Vertragsinhalt hinsichtlich der Anforderungen an diese Konformität jedoch fehlt und sich nachträglich eine Inkongruenz mit diesem außergesetzlichen normativen Umfeld zeigt. Hierin könnte eine Störung der Geschäftsgrundlage im Sinne einer Zweckstörung zu erblicken sein, bei welcher zwar der Leistungserfolg noch herbeigeführt werden kann, der Gläubiger hieran jedoch kein Interesse mehr hat. ${ }^{48}$ Problematisch erscheint diese Annahme jedoch, wenn man sich abermals vor Augen führt, dass sich die Geschäftsgrundlage aufgrund der Ambiguität der ethischen oder religiösen Normen, denen das Anlageprodukt entsprechen soll, kaum hinreichend bestimmt fassen lässt. Dieses wird im Kontext des Islamic Banking besonders deutlich, bei denen die Parteien übereinstimmend von einer Shariakonformität des Produktes ausgehen, ohne hierüber eine entsprechende Abrede getroffen zu haben. Ebenjene Konformität mit der Sharia erweist sich vor dem Hintergrund des Spektrums verschiedener Auslegungsmöglichkeiten des islamischen Rechts als rechtlich kaum handhabbare Größe. Ein Verstoß kann nur dann festgestellt werden, wenn man zugleich die von den Parteien als maßgeblich erachtete Spielart des islamischen Rechts bestimmt, was jedoch nur selten gelingen wird. Solange diese Konkretisierung unterbleibt, erscheint der Anwendungsbereich des $\$ 313$ BGB auf die äußerst seltenen Fälle beschränkt, in denen sich die fehlende Übereinstimmung mit der Sharia zweifelsfrei bestimmen lässt, etwa wenn weder die AAOIFIStandards noch irgendeine islamische Rechtsschule das Produkt als shariakonform ansieht. War dies dem Anbieter des islamischen Bankprodukts bewusst, wird jedoch regelmäßig die oben skizzierte Risikozuweisung eingreifen und eine Haftung aus culpa in contrahendo $(\mathbb{S} \mathbb{2} 280,311$ Abs. 2 BGB) eingreifen, sofern die Bank ein ersichtlich nicht mit der Sharia konformes Produkt vertrieben hat.

Ein weiterer denkbarer Anknüpfungspunkt für die Anwendung der Regeln über die Störung der Geschäftsgrundlage könnte noch darin gesehen werden, dass man weniger auf die Beeinträchtigung der ideellen als vielmehr der wirtschaftlichen Sphäre abstellt. Insofern ist es bei am Kapitalmarkt gehandelten Produkten durchaus üblich, dass der Markt auf eine Neubewertung der Übereinstimmung des Produktes mit den ethischen oder religiösen Vorgaben mit Kursabschlägen reagiert. Auch hierfür lässt sich exemplarisch das Islamic Banking mit der bereits erwähnten Shariafalle anfüh- 
ren. ${ }^{49}$ Bei einem schwerwiegenden Einbruch des Kurses erscheint es zumindest denkbar, diese als nachträgliche Äquivalenzstörung aufzufassen. Dass die Regeln über die Störung der Geschäftsgrundlage zur Anwendung kommen können, wenn die Äquivalenz von Leistung und Gegenleistung etwa aufgrund von Gesetzesänderungen nachträglich gestört ist, ist im deutschen Recht anerkannt. ${ }^{50}$ Aber auch dieser Ansatz kann letztlich nicht überzeugen, da abermals vorrangig nach der Verteilung des Kursrisikos zu fragen ist. Erwirbt der Anleger ein börsennotiertes shariakonformes Anlageprodukt, ist für den Kapitalmarkt die Volatilität der Kurse gerade ein vom Anleger in Kauf genommenes Risiko. Die Investition in ein von schwer kalkulierbaren externen Faktoren wirtschaftlich abhängiges Produkt stellt dabei unverkennbar ein gewisses Risikogeschäft dar. Lässt sich der Anleger auf dieses Risiko ein, so trifft ihn auch die Gefahr der Realisierung ebenjener Gefahr. ${ }^{51}$ Letztlich erscheint daher angesichts dieses normativen Ansatzes auch die Anknüpfung an eine nachträgliche Inäquivalenz, die auf einer Neubewertung des Produkts durch den Kapitalmarkt beruht, kein gangbarer Weg zur Anwendung der Regeln über die Störung der Geschäftsgrundlage.

\section{Zwischenfazit}

Zusammenfassend erscheint der Anwendungsbereich der Störung der Geschäftsgrundlage für religiös oder ethisch motivierte Geldanlagen äußerst begrenzt. Allenfalls kommt dieser Rechtsfigur daher eine Funktion als „Grobfilter“ für solche seltenen Fälle zu, bei denen entgegen der Erwartung der Parteien die Nichtübereinstimmung des Produktes mit den gesetzten ethischen oder religiösen Vorgaben, welche ihrerseits jedoch nicht Vertragsinhalt geworden sind, sicher festzustellen ist. Eine Anpassung des Vertrages nach $\mathbb{3} 313$ Abs. 1 BGB kommt hierbei nur dann in Betracht, wenn sich die Übereinstimmung mit den Vorgaben noch nachträglich herstellen lässt. Ist dies nicht möglich, eröffnet $\$ 313$ Abs. 3 S. 1 BGB dem Anleger das Recht zum Rücktritt, an dessen Stelle für Dauerschuldverhältnisse nach S. 2 die Kündigung tritt. $^{52}$

\section{Auslegung des Vertrages anhand außerrechtlicher Vorgaben?}

Für islamische Finanzierungsverträge ist die These vertreten worden, dass der Hinweis auf die Grundsätze der Sharia wie in der Beximco-Entscheidung (oben C.I.)

49 Zur sog. Sharia-Falle siehe auch noch unten unter E. I. und III, sowie Casper, Normgeltung und Normumgehung - Vom Zinsverbot zum Islamic Finance, in: Jansen/Oestmann, Gewohnheit, Gebot, Gesetz - Zur Entstehung von Normen in Geschichte und Gegenwart, im Erscheinen 2011, S. 301, 323 ff.. Allgemein zu diesem Problem Bälz, Sharia Risk?, Occasional Publication 9 (2008), Islamic Legal Studies Program, Harvard Law School, abrufbar unter: www.law.harvard.edu/programs/ilsp/ publications/balz.pdf (zuletzt aufgerufen am 22.5.2011).

50 Vgl. nur Bamberger/Roth/Unberath, $\$ 313$ Rn. 56.

51 So allgemein für Risikogeschäfte auch HK-BGB/Schulze, $\mathbb{3} 13$ Rn. 16.

52 Zum Verhältnis von $\mathbb{} 313$ Abs. 3 S. 2 zum ebenfalls möglichen $\mathbb{} 314$ Feldhahn, NJW 2005, 3381, $3382 \mathrm{f}$. 
zwar nicht als Rechtswahl verstanden werden könne, wohl aber als Legitimation, den Vertrag im Lichte der Sharia auszulegen. ${ }^{53}$ Als Vorbild wird auf die Rechtsprechung zu Eheverträgen verwiesen, die zwar deutschem Recht unterliegen, aber von zwei islamischen Ehepartnern in Bezug auf das islamische Eherecht geschlossen werden. ${ }^{54}$ Auch wenn eine „hybride Rechtswahl“ wie im Fall Beximco insoweit fehlt, scheint eine Auslegung im Lichte des islamischen Rechts im Wege einer teleologischen Auslegung denkbar, sofern der Wille beider Parteien ergibt, dass die Sharia zur Anwendung kommen soll. Ein deutsches Gericht wäre somit berufen, Zweifelsfragen im Lichte der Sharia auszulegen oder mögliche Lücken im Vertrag zu schließen.

Auch hinsichtlich dieses Vorschlages ist Vorsicht geboten. Abermals ist die Erkenntnis in den Mittelpunkt zu stellen, dass es "das" islamische Recht nicht gibt, sondern die Sharia von einer hohen normativen Ambiguitätstoleranz gekennzeichnet ist. ${ }^{55}$ Wollte ein deutsches Gericht im Wege der Auslegung also beurteilen, ob ein Finanzierungsvertrag und die dort verwendeten Termini wirklich dem islamischen Recht entsprechen, wäre es hierzu kaum in der Lage. Nun könnte man dem entgegenhalten, dass deutsche Gerichte bei kollisionsrechtlichen Sachverhalten oft nicht in der Lage sind, das ausländische Recht aus eigener Anschauung heraus zu beurteilen, sich aber dazu der Hilfe von Gutachtern bedienen können. Der entscheidende Unterschied wird aber sofort offenbar, wenn man sich abermals vor Augen führt, dass es hier nicht um die Auslegung im Lichte einer fremden Rechtsordnung sondern um religiöses Binnenrecht geht, das keine zentrale Autorität kennt. Jenseits der offenkundigen Verstöße wie im oben skizzierten Fall Blom vs. TID dürfte die Einholung verschiedener Rechtsgutachten durchaus zu unterschiedlichen Ergebnissen führen. Deshalb kann auch der Vergleich mit der Rechtsprechung zur Auslegung von Eheverträgen unter islamischen Ehegatte nicht ohne weiteres überzeugen. In der Entscheidung des Bundesgerichtshofs vom 14.10.1998, ${ }^{56}$ in der es um die Wirksamkeit einer Morgengabe (dem Brautgeld in der islamischen Tradition) ging, heißt es dann auch nur, dass mit der Verwendung des Terminus „Morgengabe“ gemeint sein könnte, dass „das gesamte Spektrum der Funktionen umfaßt werden sollte, die der Morgengabe in den islamischen Rechtsordnungen" ${ }^{57}$ zukommen soll. Damit wird also nicht auf „das“ islamische Recht in seiner Gesamtheit verwiesen, sondern auf die Ausprägungen, die ein aus dem islamischen Recht stammendes Rechtsinstitut in den staatlichen Rechtsordnungen einiger islamischer Staaten erfahren hat, namentlich derjenigen, der die Ehegatten bei ihrer Eheschließung angehörten. Nun könnte man ge-

53 Dies andeutend etwa Bälz, ZVglRWiss 109 (2010), 272, 286 f.

54 BGH, NJW 1999, 574, 575; vgl. zum Ganzen auch Rohe, Islamisches Recht, 2009, S. $366 \mathrm{ff}$, ohne dieses Ergebnis allerdings explizit zu befürworten.

55 Vgl. bereits oben mit Fn. 27.

56 BGH, NJW 1999, 574, 575.

57 BGH, NJW 1999, 574, 575 - Hervorhebung durch den Verf. 
neigt sein, dieser Sichtweise entgegenzuhalten, dass gerade das Erb- und Familienrecht einiger islamischer Staaten - wie in Syrien ${ }^{58}$ seinerseits auf „das“ islamische Recht verweist. Allerdings gibt es hierzu regelmäßig eine umfangreiche nationale Spruchpraxis, die durch die in dem jeweiligen Staat vorherrschende islamische Rechtsschule konkretisiert wird.

Gerade dieser Aspekt kann einen Schlüssel für die Lösung der aufgeworfenen Frage bilden. Eine Auslegung eines islamischen Finanzierungsvertrages im Lichte des islamischen Rechts ist dann denkbar, wenn sich aus dem Vertrage eine nähere Bestimmung der Spielart des islamischen Rechts ergibt. Dies kann etwa geschehen, indem der Vertrag auf die Interpretation des islamischen Rechts durch eine Rechtsschule oder durch bestimmte islamische Rechtsgelehrten oder auf die Lösung der jeweiligen Rechtsfragen durch den Sharia Board der AAOIFI bzw. des IFSB verweist. ${ }^{59}$

Der hier unterbreitete Lösungsvorschlag fordert quasi einen Bestimmtheitsgrundsatz für den Verweis auf außerrechtliche Vorgaben oder deren Inbezugnahme im Wege der Auslegung. Die Sinnhaftigkeit einer derartigen Begrenzung zeigt sich noch stärker im Bereich des Ethikbanking. Soweit die Vertragspartner eines Nachhaltigkeitsfonds nicht auf die Interpretation von Nachhaltigkeit durch eine bestimmte Organisation verweisen, würde man jeden Rechtsanwender überfordern, wenn er den Vertrag im Einklang mit dem deutschland- oder gar weltweit vorherrschenden Begriff der Nachhaltigkeit auslegen sollte.

\section{E. Risikoverteilung (die sog. Shariafalle) und Haftung}

\section{Fallgruppen und Risikoverteilung}

Mit dem Stichwort Shariafalle wird in der Islamic Finance-Szene die Situation beschrieben, dass sich bei einem schon aufgelegten, in der Regel börsennotierten islamischen Finanzprodukt während der Vertragslaufzeit herausstellt, dass es in Wirklichkeit nicht shariakonform ist. Dies führt in der Regel dazu, dass sein Börsenkurs einbricht, da es nunmehr nur noch schwer bis gar nicht mehr verkäuflich ist. Besonders anfällig hierfür sind Finanzprodukte, bei denen die Übereinstimmung mit den religiösen oder sonstigen außerrechtlichen Vorgaben schon zum Zeitpunkt der Emission nicht unumstritten war. Paradigmatisch war insoweit eine Fatwa des international renommierten islamischen Rechtsgelehrten Taqi Usmani aus dem Jahre $2008,{ }^{60}$ der auch Vorsitzender des Sharia Boards der AAOIFI ist. Es ging um die Zulässigkeit einiger islamischer Anleihen bzw. Zertifikate, den sog. Sukuk, die keine

58 Die Parteien in der Entscheidung BGH, NJW 1999, 574 waren im Zeitpunkt ihrer Eheschließung syrische Staatsangehörige.

59 Tendenziell in diesem Sinne auch noch Bälz (Fn. 23) S. 569, 580 f., 583.

60 Vgl. zu dieser Fatwa mit Begründung Usmani, Sukuk and their contemporary application, abrufbar unter: http://sukuk.me/news/articles/73/Sukuk-and-their-contemporary-applications.html (zuletzt aufgerufen am 23.5.2011). 
Verzinsung, sondern eine gewinnabhängige Vergütung vermitteln. ${ }^{61}$ Einige Emittenten dieser Anleihen versprachen ihren Gläubigern zwar keine Mindestrendite wie im Fall Blom gegen TID, jedoch garantierten sie auf Basis einer sog. Sukuk-al-Mudara$b a$ den Anlegern am Ende der Laufzeit die Rückzahlung des eingesetzten Kapitals, obwohl bei einer klassischen $M u d a r a b a^{62}$ das Verlustrisiko des investierten Kapitals für den Anleger typusbildend ist. Zwar entfaltet die Fatwa eines Rechtsgelehrten, bei der es sich um eine Art Rechtsgutachten handelt, keinerlei Bindungswirkung, ${ }^{63}$ jedoch brach der Kurs der entsprechenden Papiere aufgrund des hohen Renommees und der Autorität von Taqi Usmani ein, zumal die AAOIFI diese Ansicht alsbald in ihre Standards zur Sukuk übernahm, während sie sich vorher nicht zu dieser speziellen Frage geäußert hatte.

Will man nun einer möglichen Haftung des Emittenten nachspüren, gilt es sich zunächst nochmals die Risikoverteilung für die Fehleinschätzung der Shariakonformität ins Gedächtnis zu rufen. Insoweit ist abermals auf die oben unter C.II.2. angestellten Überlegungen zu verweisen. Danach ist der Anbieter nicht per se in der Verantwortung dafür, dass sich die Einschätzung der Shariakonformität später ändert. Vielmehr trifft ihn nur die Pflicht, keine offenkundig den allgemein akzeptierten islamischen Prinzipien widersprechende Produkte aufzulegen und für eine Zertifizierung zu sorgen. Anderenfalls hat er auf Zweifel hinzuweisen und zum Ausdruck zu bringen, dass er für die Konformität mit der Sharia keine Verantwortung übernehmen will.

Mit Blick auf mögliche Haftungsansprüche lassen sich letztlich drei Fallgruppen unterscheiden. (1) Bereits beim Vertrieb des Finanzprodukts steht fest, dass es nicht wie deklariert shariakonform ist (anfängliches Fehlen der Konformität). Entweder hat der Emittent selbst die fehlerhafte Einschätzung vorgenommen oder dem von ihm beauftragten Sharia Board ist ein Fehler unterlaufen. (2) Weiterhin ist denkbar, dass die Konformität mit der Sharia während der Vertragslaufzeit wegfällt. Ein Beispiel dafür bildet die oben skizzierte sog. Shariafalle. Daneben kann man sich vorstellen, dass der Sharia Board nachträglich die Zertifizierung entzieht, da sich die Umstände geändert haben oder der Emittent dem Rat wesentliche Informationen vorenthalten hat. Bei islamischen Fonds ist (3) die Fallgruppe denkbar, dass der Fonds gegen seine eigene, shariakonforme Anlagepolitik verstößt, also solche Unternehmensanteile in das Fondsvermögen aufnimmt, die den shariakonformen Anlagebedingungen nicht entsprechen. ${ }^{64}$

61 Vgl. zur Funktionsweise einer Sukuk etwa Müller, WM 2008, 102 ff.; Sacarcelik, SZW 2010, 10, 16 ff.; Adam/Thomas, Islamic Bonds: Your Guide to Issuing, Structuring and Investing in Sukuk, 2004.

62 Vgl. den Nachw. oben in Fn. 46.

63 Vgl. zur Funktion einer Fatwa stellvertretend für Viele Rohe, Islamisches Recht, 2009, S. 74 f.

64 Vgl. speziell zur Haftung für die fehlende Konformität mit der Sharia bei islamischen Aktienfonds auch bereits Casper, FS Schneider, 2010, S. 229, $243 \mathrm{ff}$. 
Diese verschiedenen Fallgruppen sollen abschließend kurz auf mögliche Haftungsansprüche anhand der Vorgaben im deutschen Recht durchleuchtet werden. Es wird unterstellt, dass der Emittent einen Prospekt herausgegeben hat, in dem er die Konformität mit der Sharia seines Finanzprodukts herausgestrichen hat.

\section{Anfängliches Fehlen der Konformität}

Bewirbt der Finanzdienstleister sein Produkt in einem Verkaufsprospekt als shariakonform oder preist er dies als nachhaltig bzw. ökologisch an, während dies in Wahrheit ersichtlich nicht der Fall ist, liegt es nahe, über einen Anspruch aus Prospekthaftung nachzudenken. In der Praxis wird es regelmäßig um eine Prospekthaftung nach $\$ 127$ InvG gehen. ${ }^{65}$ Dass es sich bei der Shariakonformität um Angaben handelt, die für die Beurteilung der Anteile von wesentlicher Bedeutung sind, wird man ohne weiteres annehmen können, da das Fehlen dieser Eigenschaft - wie gezeigt - erheblichen Einfluss auf die Preisbildung derartiger Papiere haben kann. Nichts anderes gilt bei Ethikfonds, bei denen sich herausstellt, dass doch in Unternehmen aus der Atomenergiebranche oder der Rüstungsindustrie investiert wurde.

Doch praktisch dürften diese Fälle nur sehr selten bleiben. Die Voraussetzung für eine derartige Prospekthaftung besteht gerade darin, dass unzweifelhaft feststeht, dass die außerrechtlichen Vorgaben nicht eingehalten werden. Diese offenkundigen Fälle werden jedoch rar gesät sein. Regelmäßig wird das Anschauungsmaterial jedoch so gestrickt sein, dass eine positive Zertifizierung durch den Sharia Board vorliegt, die auch durchaus von Teilen der islamischen Rechtswissenschaft geteilt, von anderen aber verworfen wird. Eindeutige Fälle wie Blom vs. TID, wo der Verstoß auch für den islamwissenschaftlichen Laien mit Händen zu greifen ist, dürften dagegen eher selten vorkommen. Bewegt sich der Sharia Board also in dem üblichen, vertretbaren Interpretationspluralismus, der für das islamische Recht genauso kennzeichnend ist wie für den Bereich des Ethikbankings, sprechen die besseren Gründe dafür, dem Sharia- bzw. Ethikrat eine Einschätzungsprärogative zuzubilligen. Eine Haftung ist außer bei einer offensichtlichen Unrichtigkeit der Einschätzung ${ }^{66}$ vor allem dann realistisch, wenn der Anbieter des Finanzprodukts bzw. dessen Sharia Board - quasi einer Black-Box ${ }^{67}$ vergleichbar - sein Ergebnis für die positive Zertifizierung nicht offengelegt hat und pauschal mit der Islamkonformität seines Produkts wirbt. Damit wird der Anbieter zur Transparenz verpflichtet. Ist die Zulässigkeit des angebotenen Produkts bekannterweise umstritten, ist hierauf im Prospekt

65 Bei börsennotierten Produkten wie dem Sukuk sind hingegen $\mathbb{S} 44,45$ BörsG einschlägig.

$66 \mathrm{Ob}$ in diesen Fällen der Sharia Board als Prospektverantwortlicher oder Sachverständiger wie ein Wirtschaftsprüfer aus der bürgerlich-rechtlichen Prospekthaftung in Anspruch genommen werden kann, kann an dieser Stelle nicht vertieft werden, vgl. allg. dazu etwa Assmann in: Assmann/Schütze, Handbuch des Kapitalanlagerechts, 3. Aufl. 2007, \$6 Rn. 155 ff.

67 Black-Box wird hier im Sinne des aus der Informatik bekannten Black-Box-Test verstanden und nicht als alles aufzeichnender Recorder, nach dem es nach dem Absturz zu suchen gilt. 
hinzuweisen oder dies in den dem Anlagepublikum zugänglich zu machenden Fat$w a$ 's des Sharia Boards offenzulegen. Denkbar wäre eine Haftung eventuell auch noch dann, wenn der Anbieter in seinem Prospekt nicht darauf hinweist, dass sich seine Einschätzung von Konformität mit der Sharia nicht mit den Anforderungen der beiden großen Nichtregierungsorganisationen im Bereich des Islamic Finance, der AAOIFI in Bahrain bzw. dem IFSB ${ }^{68}$ deckt. Zumindest einstweilen scheint mir eine Haftung in dieser Konstellation aber nicht zwingend, da die beiden Organisationen noch nicht allgemein anerkannt sind.

Jenseits der spezialgesetzlichen Prospekthaftung ist eine Haftung aus culpa in contrahendo oder ausnahmsweise auch aus bürgerlich-rechtlicher Prospekthaftung zu erwägen. Letzteres setzt aber voraus, dass die gesetzlichen Prospekthaftungsansprüche nicht anwendbar sind, aber gleichwohl ein Prospekt oder eine entsprechende Darstellung und nicht nur eine Werbebroschüre beim Vertrieb eingesetzt wurde. ${ }^{69}$

\section{Nachträglicher Wegfall der Konformität}

Eine Haftung beim nachträglichen Wegfall der Konformität mit den religiösen oder außerrechtlichen Vorgaben kommt ebenfalls nicht ohne weiteres in Betracht. Mögliche Ansatzpunkte sind eine Verletzung der Informationspflicht bei Entzug der Zertifizierung bzw. eine unterlassene Anpassung, derart, dass die Konformität wiederhergestellt wird.

Das Paradebeispiel für eine Haftung wegen der Verletzung einer Informationspflicht bildet der Entzug der Zertifizierung durch den Sharia Board während der Vertragslaufzeit. Gegen eine Informationspflicht könnte man geneigt sein, die fehlende Verbindlichkeit der Entscheidungen des Sharia Boards ins Feld zu führen. Nur ausnahmsweise wird sich der Anbieter der Finanzprodukte vertraglich verpflichtet haben, seine Anleger über Änderungen der Entscheidungen des Sharia Boards zu informieren. Aufgrund der zentralen Bedeutung der Zertifizierung für die Anlageentscheidung des Investors wird man eine Aufklärungspflicht jedoch grundsätzlich bejahen können. Fraglich ist allerdings, ob auch jenseits der Ad-hoc-Publizitätspflicht iSd. $\$ 15 \mathrm{WpHG}$ eine Pflicht zur unterjährigen Aufklärung besteht. ${ }^{70}$ Zumindest soweit die islamischen Finanzprodukte fungibel sind, liegt eine Pflicht zur sofortigen Information der Anleger nahe, wobei auf den Unverzüglichkeitsmaßstab des $\mathbb{1 2 1}$ Abs. 1 S. 1 BGB zurückgriffen werden kann.

68 Vgl. oben Fn. 44.

69 Vgl. allgemein zum verbleibenden Anwendungsbereich der auf die Rechtsprechung zurückgehenden bürgerlich-rechtlichen Prospekthaftung neben den gesetzlichen Ansprüchen in $\mathbb{\int} \mathbb{S 4} 445$ BörsG,

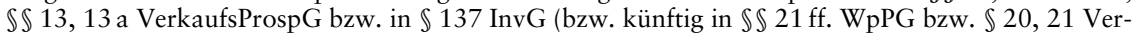
$\mathrm{mAnlG}$ ) etwa Assmann in: Assmann/Schütze (Fn. 65), $\mathbb{S} 6 \mathrm{Rn} .129 \mathrm{ff}$.

70 Vgl. dazu näher Casper, FS Schneider, 2010, S. 229, 244. 
Eine Pflicht zur Anpassung des Finanzprodukts, sodass es künftig wieder den Vorgaben des islamischen Rechts oder der sonstigen außerrechtlichen Vorgaben genügt, kommt regelmäßig bereits aus tatsächlichen Gründen nicht in Betracht, da dies fast durchweg eine grundlegende Vertragsänderung voraussetzen würde oder oftmals schlicht unmöglich ist. Eine abweichende Situation ergibt sich bei Ethikfonds oder islamkonformen Fonds, sofern die Anleihebedingungen eine entsprechende Anpassungspflicht vorsehen. ${ }^{71}$ Ein Verstoß gegen die Anpassungspflicht löst dann einen auf $\mathbb{S} \$ 280 \mathrm{ff}$. BGB gestützten Schadensersatzanspruch aus.

\section{Verstoß gegen Anlagegrundsätze}

Weitgehend unproblematisch ist schließlich die letzte Fallgruppe, die nur bei Fonds vorkommt. Es geht darum, dass der Fonds sich nicht an die von ihm selbst in den Anlagebedingungen aufgestellte Anlagepolitik hält, mit der man eine Vereinbarkeit mit den religiösen oder außerrechtlichen Vorgaben zu erreichen sucht. Dieser Verstoß gegen die Vertragsbedingungen beinhaltet zugleich eine Verletzung des Investmentvertrages, was wiederum eine Haftung nach $\$ 280$ Abs. 1 BGB auslöst.

Die Schwierigkeiten liegen jedoch auf der Rechtsfolgenebene. Unklar ist häufig, worin der Schaden des Anlegers bestehen soll. Eine Rückabwicklung des Vertrages wird regelmäßig nicht in Betracht kommen, da der spätere Verstoß nicht für die Kaufentscheidung kausal war. Eine Ausnahme gilt nur insoweit, als auch zugleich ein Verstoß gegen den Prospekt vorliegt, was jedoch selten sein dürfte, und sofern dieser Anspruch auf Prospekthaftung, der eine Naturalrestitution ermöglicht, noch nicht verjährt ist.

\section{Die Problematik sog. Haftungsfreizeichnungsklauseln}

Zumindest bei islamkonformen Fonds ist zunehmend die Tendenz zu beobachten, dass sich die Anbieter versuchen, von der Haftung im oben skizzierten Sinne soweit wie möglich freizuzeichnen. Ein aktuelles Beispiel bildet der in Luxemburg aufgelegte Meridio Islamic Funds, ein Aktienfonds, der auch in Deutschland vertrieben wird. In dem Verkaufsprospekt heißt es zunächst: „Die Überprüfung der Konformität ... mit der Sharî‘a ... wird von einem unabhängigen Sharî‘a-Board sowie optional zusätzlich vom Sharı̂‘a-Berater durchgeführt“.${ }^{72}$ Mag sich der Anleger hierdurch noch in Sicherheit wähnen, erfährt er nun alsbald: „Es wird davon ausgegangen, dass sich Anleger und Interessenten eigenverantwortlich informieren und aufgrund der Aussage eigener Sharî‘a-Berater oder ähnlich verlässlicher Quellen davon überzeugen, dass die Gesellschaft oder der jeweilige Teilfonds nicht gegen Sharî‘a-Grundsätze

$71 \mathrm{Zu}$ den Einzelheiten vgl. Casper, FS Schneider, 2010, S. 229, 244.

72 Verkaufsprospekt Meridio Islamic Funds - Meridio Global Islamic Multi Asset -, Stand Januar 2010, S. 11; ebenso Vereinfachter Verkaufsprospekt des Allianz Global Investors Islamic Fund v. 17.1.2009, S. $6 \mathrm{f}$. 
verstößt. Es besteht ... das Risiko, dass sich der Status der Sharî‘a-Konformität im Laufe der Zeit ändert. Die Gesellschaft übernimmt keine Haftung bezüglich dieser Änderung “. ${ }^{73}$

Eine derartige Klausel zielt vor allem auf die zweite Fallgruppe, der nachträglichen Änderung der Einschätzung der Konformität mit der Sharia. Ein Anschluss der Haftung für fehlerhafte Prospekte (erste Fallgruppe) im Voraus scheitert bereits an $\mathbb{} 47$ BörsG. ${ }^{74}$ Für die Frage, ob die Haftungsfreizeichnung für den nachträglichen Wegfall der Konformität zulässig ist, ist man geneigt, auf $\mathbb{} 307$ Abs. 2 BGB zuzusteuern. Dabei ist aber vorab danach zu fragen, inwieweit die Verantwortlichkeit des Anbieters überhaupt reicht, da nur in diesen Fällen wirklich eine Haftungsfreizeichnung im Rechtssinne vorliegt. Damit schließt sich der Kreis zu den oben unter C.II.2. angestellten Überlegungen zur Risikoverteilung. Dort war hervorgehoben worden, dass es gerade die Aufgabe der Bank ist, entweder darauf hinzuweisen, dass sie die Konformität mit der Sharia nicht überprüft, oder dies mittels der Zertifizierung durch einen Sharia Board sicherzustellen. Genau diesen Anforderungen kommt der Meridio Islamic Funds im obigen Beispiel nach. Zum einen lässt er durch einen Sharia Board seine Fonds zertifizieren, zum anderen weist er seine Kunden darauf hin, dass er für die Richtigkeit von dessen Entscheidung keine Gewähr übernehmen will. All dies ist noch keine Haftungsfreizeichnung im engeren Sinne. Eine wirkliche Freizeichnung von der Haftung liegt allenfalls in dem letzten Satz, dass die Gesellschaft für Fehleinschätzungen durch den von ihr eingesetzten Sharia Board keine Haftung übernehmen will, obwohl der Anbieter hiermit geworben hat. ${ }^{75}$ Eine derartige Haftungsfreizeichnung ist an $\$ 307$ Abs. 2 BGB zu messen. Sie hält einer Inhaltskontrolle jedoch Stand, da der Anbieter genau dem nachgekommen ist, wozu er nach der hier entwickelten Risikoverteilung verpflichtet ist, nämlich darauf hinzuweisen, dass auch die Zertifizierung durch einen Sharia Board keine vollständige Rechtssicherheit bringt. Sofern der Sharia Board mit qualifizierten Personen besetzt ist und der Anbieter zum Ausdruck bringt, dass er für die Richtigkeit von dessen Rechtsansichten mit Blick auf die vielfältigen Strömungen im Islam nicht einstehen will, ist eine derartige Haftungsfreizeichnung nicht zu beanstanden.

Von seinen Informationspflichten und der Pflicht, keine Produkte anzubieten, die offenkundig die außerrechtlichen Vorgaben verfehlen, kann sich der Anbieter nicht

73 Verkaufsprospekt Meridio Islamic Funds - Meridio Global Islamic Multi Asset -, Stand Januar 2010, S. 11.

74 Auch wenn $\$ 127$ InvG keine entsprechende Regelung bereithält, gilt dort nach der zutreffenden Auffassung unter Rückgriff auf $\$ 307$ Abs. 2 Nr. 1 BGB nichts anderes, vgl. nur Köndgen in Berger/Steck/ Lübbenhüsen, InvG, InVStG, 2009, $\$ 127$ InvG Rn. 24 m. weit. Nachw.

75 Eine Haftungsfreizeichnung im engeren Sinne liegt also nur hinsichtlich einer möglichen Haftung vor, die daran anknüpft, dass der Anbieter mit der nicht offenkundigen Fehleinschätzung durch den Sharia Board gewoben hat. 
freizeichnen, da es sich insoweit um eine Kardinalpflicht des Finanzierungsvertrages handelt.

\section{F. Zusammenfassendes Fazit}

Der vorstehende Beitrag ist mit dem Anspruch angetreten, Unterschiede und Gemeinsamkeiten von Islamic Banking und Ethikbanking aufzuzeigen. Dabei musste angesichts des rechtstatsächlichen Anschauungsmaterials der Schwerpunkt im Zusammenhang mit dem Islamic Banking gesetzt werden. Es konnte jedoch gezeigt werden, dass sich beim Ethikbanking ganz ähnliche Probleme stellen. Diese sind teilweise sogar noch gravierender. Eine der zentralen Unsicherheiten des Islamic Finance ist die normative Ambiguitätstoleranz des islamischen Rechts. Genau dieses Problem stellt sich aber auch, wenn man seine Finanzprodukte an Maßstäben wie „ökologisch“, „ethisch“, „nachhaltig“ oder dergleichen mehr ausrichtet. Man kann sogar die Behauptung wagen, dass die Maßstäbe in diesem Kontext noch viel unsicherer und interpretationsbedürftiger sind.

Die wesentlichen Ergebnisse zur Risikoverteilung, Geschäftsgrundlage, Auslegung und Haftung lassen sich in acht Leitsätzen zusammenfassen.

1. Die Orientierung eines Finanzierungsvertrages an außerrechtlichen Vorgaben wie dem islamischen Recht oder ethischen bzw. sonstigen religiösen oder weltanschaulichen Vorgaben führt regelmäßig nicht zur Anwendung des $\$ 313$ BGB.

2. Vielmehr lässt sich meist bereits im Wege der Vertragsauslegung ermitteln, wie das Risiko einer Verfehlung dieser außerrechtlichen Vorgaben zwischen den Parteien sachgerecht zu verteilen ist. Dabei ist es nicht angängig, dieses Risiko allein einer Vertragspartei aufzubürden.

3. Die Bank bzw. der Finanzdienstleister ist dafür verantwortlich, dass er seine Produkte nur dann mit dem Siegel „shariakonform“, „nachhaltig“, „ökologisch“ oder dergleichen versieht, wenn sichergestellt ist, dass diese Vorgaben nicht offenkundig verfehlt werden. Insoweit trifft den Anbieter eine Informationslast.

4. In dem breiten Bereich des Interpretationsspielraums, was im Einzelfall letztlich noch den religiösen oder ethischen Vorgaben entspricht, trifft den Anbieter hingegen nur die Pflicht, für Klarheit über das verbleibende Restrisiko einer Fehleinschätzung Sorge zu tragen. Entweder muss er den Kunden hinweisen, dass er hierfür keine Garantie übernimmt oder er muss eine Zertifizierung durch eine anerkannte Organisation bzw. externe Gutachter sicherstellen. Für deren Fehleinschätzung haftet der Finanzdienstleister aber nicht. Dieses Risiko ist vielmehr dem Anleger zuzuweisen, den aufgrund seines Wunsches, ein Finanzprodukt, das vagen religiösen oder ethischen Vorgaben entsprechen soll, ebenfalls eine Einschätzungspflicht trifft. 
5. Eine Auslegung eines islamischen Finanzierungsvertrages im Lichte des islamischen Rechts ist nur dann denkbar, wenn sich aus dem Vertrag eine nähere Bestimmung der Ausprägung des islamischen Rechts ergibt. Dies kann etwa geschehen, indem der Vertrag auf die Interpretation des islamischen Rechts durch eine Rechtsschule oder bestimmte islamische Rechtsgelehrten oder auf die Lösung der jeweiligen Rechtsfragen durch den Sharia Board der AAOIFI bzw. des IFSB verweist.

6. Ist bereits bei Emission eines Finanzprodukts eine Konformität mit den außerrechtlichen Vorgaben nicht gegeben, kommt eine Prospekthaftung oder jenseits davon ein Anspruch aus c.i.c. in Betracht. Dies setzt jedoch voraus, dass die fehlende Übereinstimmung für den Emittenten offenkundig war. In den praktisch relevanten Fällen einer Fehleinschätzung durch den Sharia- oder Ethikrat ist eine Haftung des Finanzdienstleisters grundsätzlich zu verneinen.

7. Eine Haftung des Anbieters für den nachträglichen Wegfall der Konformität ist regelmäßig nicht gegeben, sofern dieser nicht vorhersehbar war. Allerdings besteht zumindest bei fungiblen Produkten eine Pflicht zur unverzüglichen Information des Anlegers, deren Verletzung ihrerseits schadensersatzbewährt ist.

8. Eine Haftungsfreizeichnung liegt nur dann vor, wenn die Parteien von der in den Leitsätzen 2 bis 4 aufgestellten Risikoverteilung vertraglich abweichen, nicht hingegen, wenn „Haftungsfreizeichnungsklauseln“ diese Risikoverteilung nur klarstellen. Eine Haftungsfreizeichnung für eine Fehleinschätzung durch den Sharia- oder Ethikrat ist jenseits der Fälle der Offenkundigkeit zulässig, soweit man dieses Risiko einer Fehleinschätzung nicht schon im Wege der Vertragsauslegung dem Anleger zuweist. 